\title{
Oportunidades de participación en las políticas de servicios sociales municipales en España
}

\author{
Opportunities for participation in the policies of municipal \\ social services in Spain
}

\author{
Enrique Pastor-Seller / epastor@um.es \\ Universidad de Murcia, España
}

\begin{abstract}
The article presents an analysis and systematic evaluation of the opportunities for citizen participation in the public policies of social welfare at the local level in Spain and their actual impact on the design, implementation, execution and evaluation of public policies of social welfare at the municipal level. The results of the investigation, between 2004 and 2012, permit, first, to present the type of offer diverse opportunities for participation in the regional level of social welfare in Spain and then identity, from its participants, dimensions that help to improve efficiency of public welfare policies at the municipal level by intensifying processes of democratization. The results show little opportunity to participate and if more formal than real, not to favor the possibility of an exercise of power or actual impact on the design, implementation and evaluation of municipal social policies.
\end{abstract}

Key words: quality of democracy, public policy, participation, analysis and evaluation, welfare policies.

Resumen: El artículo presenta un análisis y evaluación sistemática de las oportunidades de participación ciudadana en las políticas públicas de bienestar social en el ámbito local en España y su impacto efectivo en el diseño, implementación, ejecución y evaluación de las políticas públicas de bienestar social en el ámbito municipal. Los resultados de la investigación continua, entre 2004-2012, permite, en primer lugar, presentar la diversa tipología de oferta de oportunidades de participación en el ámbito autonómico de bienestar social de España y, posteriormente, identificar, a partir de sus participantes, las dimensiones que contribuirían a dotar de mayor eficacia las políticas públicas de bienestar en el ámbito municipal, intensificando procesos democratizadores. Sus resultados nos muestran escasas oportunidades de participación y, en su caso, más formales que reales, al no favorecer la posibilidad de realizar un ejercicio de poder o impacto efectivo en el diseño, ejecución y evaluación de las políticas sociales municipales.

Palabras clave: calidad democrática, políticas públicas, participación, análisis y evaluación, políticas de bienestar. 


\section{Presentación}

El artículo presenta un análisis y evaluación de la oferta de oportunidades de participación ciudadana en el diseño, implementación, seguimiento y evaluación de las políticas públicas de servicios sociales municipales en España y su potencial influencia en la agenda y gestión de las políticas públicas de bienestar social local. Los resultados de la investigación permiten, en primer lugar, sistematizar a modo de tipologías la oferta de mecanismos, órganos y procesos de participación en el diseño, gestión y evaluación de los servicios sociales municipales y, posteriormente, identificar las dimensiones de análisis que contribuirían a dotar de mayor eficacia a las políticas públicas sociales, mediante una intensificación de la participación ciudadana y la gobernanza local.

Investigar acerca de la evaluación del impacto de los mecanismos de participación en la construcción de la agenda de las políticas públicas y su incidencia en la gestión de éstas supone responder a un asunto innovador de interés preferente en el ámbito de las administraciones públicas, académicas, investigadoras y profesionales. En la actualidad emerge una progresiva demanda por la democracia directa y la participación ciudadana en la gestión pública, convirtiéndose en un asunto central y de actualidad en el debate político, civil, profesional y académico en general, y con un significado sustantivo en los políticos, investigadores y profesionales vinculados (directa o indirectamente) con las políticas sociales. La complejidad e interdependencia de los fenómenos y hechos sociales, así como las situaciones de dificultad que atraviesan personas, familias, grupos y comunidades requiere de compromisos, competencias e interacciones de los diferentes actores sociales (públicos y cívicos), convirtiéndose la participación en un elemento consustancial en este nuevo contexto pluralista y relacional.

La participación, como señalan distintos autores (Cunill, 1991, 1997; Maiz, 2000; Held, 2001; Warren, 2001; Montero, Font y Torcal, 2006; Bloundiaux, 2008; Pares, 2009, Pastor, 2011, entre otros), aporta sustantivos beneficios a la dinámica organizacional y comunitaria al proporcionar una progresiva adecuación del funcionamiento de las instituciones, romper la apatía y desconfianza ciudadana, ofrecer a los representantes herramientas para evaluar y mejorar la gestión de los asuntos públicos, permitir a la ciudadanía reconquistar y recuperar el espacio público, generar capital social, potenciar sentimientos comunitarios; permitir que la "política se socialice" y reforzar las decisiones a adoptar o, incluso, adoptadas. De ahí que la participación se haya convertido en un asunto de interés preferente y transversal 
en la agenda de los gobiernos y profesionales que desean implementar una gestión política y/o técnica eficaz, orientada a mejorar el bienestar social y la calidad de vida de la ciudadanía y de los usuarios de centros/servicios. La participación en la elaboración de las políticas sociales es "una herramienta fundamental para mejorar el diseño, evaluar los servicios y detectar las necesidades reales de la población" (Subirats, 2007: 57). Pero esta centralidad requiere de instrumentos que permitan una adecuada y transparente canalización de la misma y de procesos de decisión y gestión descentralizada, colegiada, ágil y corresponsable en los asuntos públicos sociales municipales.

La extensión y diversificación en la oferta de participación produce cierto efecto movilizador en el sentido de que los ciudadanos con similares orientaciones hacia la democracia local (Navarro, 2011) y/u organizacional, se implican más cuando existen más oportunidades, cuando viven en un contexto participativo, proporcionando así un aumento del ejercicio de ciudadanía activa. De ahí que el reto sea crear condiciones y espacios de participación/implicación ciudadana que generen oportunidades reales y favorables para la deliberación y construcción colectiva de las políticas sociales, a partir de la conformación de preferencias sólidas e informadas entre la ciudadanía en el complejo universo relacional.

Como señalan Sauca y Wences (2009), la inapelable consolidación de la participación de los ciudadanos en la toma y control de las decisiones gana terreno todos los días en las democracias occidentales; en el campo de las prácticas políticas los instrumentos que pretenden dar cuerpo a este imperativo participativo se multiplican, y en muchas sociedades se extienden a todas las escalas del gobierno (Blondiaux, 2008). Es evidente, la relevancia de la participación ciudadana en la consolidación de las democracias representativas, en tanto que el afianzamiento de esta forma de gobierno ya no depende sólo de que los ciudadanos ejerzan libremente sus derechos políticos, sino de que también éstos se involucren (participen) activamente en los diferentes ámbitos y etapas del quehacer público (Vallespín, 2000; Giddens, 2000; Bobbio, 2003; Espinosa, 2009), encontrándonos ante, según algunos autores consideran, un "giro deliberativo" (Chambers, 2003; Jorba, 2009).

\section{Metodología que orienta la investigación}

El estudio, cuyos resultados a continuación se presentan, realizado entre 2004-2012 ha permitido disponer de una tipología de los mecanismos, organismos y procesos de participación ciudadana en el sistema autonómico de servicios sociales de España, un análisis comparativo de casos, así como 
evaluar el impacto, en términos de contribuciones y potencialidades de los Consejos en la profundización democrática de las políticas públicas locales en materia de Bienestar Social y la mejora en la eficacia de la gestión de los servicios y prestaciones sociales.

El contexto de investigación ha sido el Sistema Autonómico de Servicios Sociales en España y más concretamente la oferta de oportunidades de participación asociativa y/o individual en la implementación, gestión y evaluación de las políticas sociales municipales. Para ello se ha realizado un análisis riguroso y sistemático de las últimas 17 Leyes de Servicios Sociales promulgadas y en los mecanismos y organismos que en las mismas se establecen para promover la participación en la gestión de las políticas públicas en la materia, utilizando para ello el análisis de contenido normativo.

Una vez analizada y comparada dicha tipología, se ha hecho un acercamiento a los actores participantes en los Consejos e Institutos de participación en las políticas de bienestar social general en el ámbito territorial de España, específicamente en la Región de Murcia. Para este análisis se ha efectuado un acercamiento a todas las Corporaciones Locales (Ayuntamientos y Mancomunidades) con competencias en materia de Servicios de Bienestar Social existentes en la Región de Murcia, concretamente 45 Ayuntamientos y 5 Mancomunidades, siendo las unidades de observación los mecanismos de participación implementados en éstos, con especial énfasis en los Consejos e Institutos de carácter general-territorial.

En su proceso de desarrollo, en primer lugar, se identificaron los Centros de Servicios Sociales de la región y su ámbito de actuación, entrevistando a 50 responsables técnicos del conjunto de Ayuntamientos y Mancomunidades con competencias en la materia existentes en la región, representando al universo de unidades de observación. A continuación, se aplicaron 22 entrevistas en profundidad con actores participantes en dichos mecanismos de participación, representando a un total de 22 entidades. Los actores fueron entrevistados en varias ocasiones, siendo seleccionados atendiendo a la composición de los mecanismos de participación (políticos, profesionales, organizaciones sociales y vecinales).

Atendiendo a la máxima representatividad de los discursos existentes, se contempló la participación de la diversidad de actores en los mecanismos de participación objeto de estudio, concretamente los agentes: políticos (tanto del gobierno como de los diferentes partidos políticos de la oposición), técnicos (relevancia, heterogeneidad, responsabilidad e implicación directa) y asociativos (cualificación y contraste de discursos, diversidad, máxima representatividad y permanencia como vocal en el mecanismo participativo). 
El informe final obtenido del proceso de análisis de contenido fue presentado a dos grupos de discusión formados por actores cualificados (políticos, profesionales y organizaciones sociales) distintos a los entrevistados. Este proceso de investigación y análisis ha permitido disponer de una información validada, fiable y éticamente contrastable acerca de las dimensiones que contribuirían a mejorar las políticas públicas de bienestar social local.

El enfoque metodológico empleado se ha basado en la utilización de técnicas, fundamentalmente - no en exclusiva- de carácter cualitativo, lo que ha permitido recoger los discursos, las opiniones, voces, sugerencias e interpretaciones de los protagonistas en los procesos de participación ciudadana en materia de las políticas sociales en el ámbito local. El estudio de casos, específicamente de los Consejos Municipales de Bienestar Social, ha permitido la proximidad y participación de los actores que construyen la realidad, superando, de esta forma, meras intenciones descriptivas o contables para explicar los motivos que conducen a una realidad determinada. A su vez, este método ha permitido producir conocimientos relevantes a partir de la combinación de técnicas y desde un contexto participativo de los sujetos/actores.

Considerando la complejidad del fenómeno a conocer, se han empleado diversas técnicas para la recogida de datos con el fin de incrementar la eficiencia de las mismas y solucionar el problema que representaba el análisis de la complejidad causal, fundamentalmente, la entrevista a responsables/ técnicos de todas las Corporaciones Locales de la Región, entrevistas en profundidad a los actores participantes y grupos de discusión con participantes estratégicos. De esta forma, se ha desarrollado una estrategia de triangulación metodológica - "within-method triangulation"- con el objeto de aumentar la capacidad analítica, fiabilidad de los resultados y validez en la investigación, evitando sesgos metodológicos. Para conseguir la necesaria estimación para la validez se han tenido presente los procesos más usuales, como son la triangulación de técnicas, saturación de pruebas y evidencias y contraste con investigaciones similares.

Para su contraste y ampliación se emplearon la recopilación, síntesis y análisis documental (teórico, normativo y empírico) en una doble dirección: interna y externa a las unidades de observación. En cuanto a la primera, a través de "documentos" producidos por los actores participantes, tales como: reglamentos, estatutos, actas, órdenes del día, convocatorias, etc.; respecto a la externa, orientada al análisis teórico, normativo y bibliográfico de los discursos existentes sobre la participación ciudadana, especialmente vinculada con la política social municipal, así como respecto de experiencias similares acontecidas tanto en el ámbito internacional como nacional. Todo ello ha 
permitido el contraste de los datos obtenidos en las entrevistas y los grupos de discusión con aquellos derivados del análisis documental y de contenido; desde una aproximación comprensiva y reflexiva por parte de los actores de la participación ciudadana en la gestión de las políticas sociales en el ámbito local de la Región de Murcia.

\section{Gobernanza de las políticas públicas sociales en el ámbito municipal}

En la actual sociedad del conocimiento disminuye la disposición a aceptar las decisiones adoptadas de manera jerárquica o poco transparente. Se demandan, por el contrario, nuevas formas de participación y comunicación. Gobernar ya no puede ser una acción unidireccional y jerárquica desde los poderes públicos hacia los ciudadanos y el tejido social. Gobernar requiere cada vez más capacidad de implicación y compromiso, tanto en la definición de problemas y políticas, como en la gestión de centros, servicios y programas.

El nivel local es un ámbito experimental para probar nuevos procedimientos de cooperación, así como formas innovadoras de articular liderazgo político y participación social (inteligencia cooperativa). "La sociedad del conocimiento aporta una nueva noción de ciudadanía que fusiona hermenéuticamente los diversos horizontes de significatividad; logra una comprensión empática que afirma la originalidad, autenticidad y peculiaridad social y preserva la identidad individual" (Aguirre, 2009: 235).

Las novedades y debates en torno al municipalismo se concretan en el tránsito desde el tradicional gobierno local hasta la actual "governance" local -gobierno en red o gobierno/administración/municipio relacional-. El contexto municipal proporciona un ámbito privilegiado para revitalizar la democracia, es donde encontramos los primeros y mejores ejemplos de las nuevas formas de entender la gobernación de los asuntos públicos desde el modelo de municipio relacional, caracterizado y legitimado por su capacidad de crear e impulsar redes, estimular la participación de la sociedad civil y ejercer su liderazgo gubernamental (representatividad), desde un nuevo modelo de gestión denominada "ciudadanista", abierto y democrático.

Este modelo de "hacer política" y gestionar los asuntos públicos, junto con la proximidad local, favorece construir identidades/pertenencias comunitarias, sentirnos implicados en los conflictos, en las necesidades y en la convivencia común y, por tanto, en las alternativas y decisiones finalmente adoptadas. Los gobiernos locales ya no dialogan con una comunidad homogénea, sino con múltiples comunidades e identidades que conviven y/o se interrelacionan en, desde y con el territorio. Por otra parte, la ciudadanía exige calidad 
y eficacia en la prestación de los servicios públicos, pero también participar en la misma definición y articulación de las políticas públicas que orientan el desarrollo local. De ahí que el reto sea crear condiciones y espacios de participación/implicación ciudadana que generen oportunidades reales y favorables para la deliberación y construcción colectiva de las políticas sociales, a partir de la conformación de preferencias sólidas e informadas entre la ciudadanía en el complejo universo relacional local.

La cuestión estriba en torno a cómo debe participar la ciudadanía en la definición del modelo de ciudad y de su territorio. La mayor riqueza de nuestras ciudades, pueblos, barrios, etc., es precisamente la calidad de su espacio público, como ámbito para el ejercicio de la ciudadanía, donde se acredita la capacidad de las democracias para configurar espacios de legitimidad, participación y responsabilidad ("governance capacity"). Esto es así porque las ciudades son lugares del diálogo y del conflicto, un "espacio" con infraestructura "hard" (estructural/tangible) y dinámica "soft" (relacional).

El gobierno local se nos presenta como un escenario privilegiado de participación, siendo especialmente visibles la emergencia de los espacios / mecanismos participativos en este ámbito. Así, la mayor parte de los gobiernos locales europeos se hallan, como señalan diferentes investigaciones y autores (Löffler, 2004; Hamzaoui, 2006; Alguacil, 2008; Colino y Del Pino, 2008; Ganuza y Frances, 2008; Navarro, Cuesta y Font, 2009; Sintomer y Ganuza, 2011; Putnam, 2011; Amnistía Internacional, entre otros) desde hace al menos dos decenios, inmersos en procesos de reforma.

Los objetivos que se han perseguido con estas reformas pueden sintetizarse en dos: por una parte, las administrativas, orientadas a lograr la eficiencia, la eficacia y la calidad de las estructuras administrativas locales y sus resultados en su relación con los deseos ciudadanos y, por otra, las políticas pretenden lograr el enriquecimiento de la democracia local, normalmente en forma de mayor transparencia y acceso de la ciudadanía a las decisiones públicas que les afectan. En ambos aspectos el gobierno electrónico (Naser y Concha, 2011) está jugando un papel esencial en la transparencia, rendición de cuentas y participación ciudadana.

La ciudadanía "reclama" una nueva "forma" de gobernar y de relación e influencia recíproca y continua entre administración local y ciudadanía. Una profundización democrática, entendida como ampliación, en términos cuantitativos y cualitativos, tanto de los actores (número y pluralidad representativa) que pueden intervenir en los procesos participativos, como de las cuestiones y los niveles de influencia y producción de políticas sociales por 
parte de los diferentes actores implicados (políticos, organizaciones, técnicos y ciudadanos). De ahí el indiscutible crecimiento de las experiencias participativas y de las políticas orientadas a impulsar la participación de la ciudadanía en los asuntos públicos, especialmente en el ámbito local.

Una progresiva y evidente renovación y adaptación de las estructuras democráticas que por medio de la participación e implicación más directa de la ciudadanía contribuye a mejorar la democracia (Pasquino, 1999), fomentar una mayor transparencia, legitimidad, eficacia, eficiencia e influencia en las decisiones públicas; optimizar el rendimiento institucional y formar a mejores ciudadanos y políticos.

Pero esta progresiva oferta de oportunidades de participación en las políticas públicas convive con contextos políticos e institucionales reticentes al impulso de políticas orientadas a promover la participación y la apertura de nuevos espacios y procesos participativos, y prefieren circunscribirse a mecanismos tradicionales de la democracia representativa.

Disponer de ciudadanía en mayúsculas no sólo mejora el funcionamiento de los instrumentos participativos sino el conjunto de la comunidad. De esta forma, la participación es un factor clave y materia prima para desarrollar el capital social y la inteligencia cooperativa, al servir no sólo para facilitar la prestación de determinados servicios o para legitimar determinadas decisiones y políticas, sino para promocionar conductas y actitudes ciudadanas democráticas y solidarias.

\section{La participación en contextos de fractura y exclusión social: de la participación "enjaulada" a la reivindicación en performances}

El complejo, heterogeneidad y dinámico escenario de mecanismos y prácticas participativas existentes en el ámbito municipal suelen distinguirse en torno a mecanismos de base asociativa (Consejos Municipales de participación ciudadana; Consejos Territoriales o Sectoriales; Comités de usuarios de los servicios y Agrupaciones de Desarrollo Local...); procesos y prácticas directas o deliberativos (presupuestos participativos; jurados ciudadanos o núcleos de intervención participativa; asambleas de ciudadanos; reuniones vecinales, comunales o de servicios; referéndum o consulta popular; manifestaciones, huelgas, boicots, encuestas de satisfacción; encuestas deliberativas; grupos de discusión...) y mixtos (planes estratégicos territoriales; agendas 21 ; consejos, foros o asambleas territoriales, sectoriales o de servicios; plataformas ciudadanas; talleres de prospectiva de territorios y servicios, entre otros). 
Del análisis de las investigaciones sobre participación en el ámbito local (Font, 2001; Gutiérrez, 2005; Rodríguez et al., 2005; Montero, Font y Torcal, 2006; Harms y Pereyra, 2006; INAP, 2008; Navarro, 2008, 2011; Pastor, 2011; entre otros), podemos constatar dos fenómenos: por una parte, la descentralización del Estado de Bienestar a partir de mediados de los ochenta ha provocado que los gobiernos municipales hayan tenido que buscar interlocutores sociales: tercer sector/sistema -e incluso mercantiles- en relación con sus nuevas competencias; $y$, por otra, que desarrollen estrategias adaptativas respecto a los rasgos estables y dinámicos de su estructura política en su trayectoria histórica.

De ahí que la oferta de oportunidades de participación política que impulsan, inicialmente, los Ayuntamientos, sobre todo urbanos (Navarro, 2008), se basen en el modelo tradicional y típicamente asociativo (Consejos Territoriales y Sectoriales), aunque cada vez más se están poniendo en marcha mecanismos de participación directa - modelo de orientación "ciudadanista” - o de información y/o consulta (encuestas, defensor del ciudadano, referéndum, Internet) y/o deliberativa, que suponen un proceso de discusión pública en torno a decisiones y/o iniciativas concretas (presupuestos participativos, Consejos Ciudadanos).

Estos mecanismos de participación se encuentran en la actualidad con cierto declive ante la emergencia de situaciones que "convulsionan" a la ciudadanía ante el cúmulo de desigualdades y "atentados" a los derechos sociales. Ahora observamos con nitidez acciones colectivas de protesta motivadas por las crecientes situaciones de riesgo de pobreza y exclusión social (Pastor y Sánchez, 2014) por motivos económicos, desigualdad social, crisis económica, desempleo, precariedad laboral, acumulación capitalista, elevado endeudamiento familiar, etcétera.

Protestas que, mediante dramatización, hacen visibles la desigual distribución de la riqueza, la pérdida de derechos sociales y laborales, la reducción de los servicios y prestaciones públicas, en definitiva: en un complejo, aunque construido de manera premeditada desde instancias políticas y económicas, mosaico de contradicciones y antagonismos existentes, que con toda probabilidad derivarán en la ruptura de la cohesión y la paz social, y cuya orientación es sensibilizar a la ciudadanía acerca de una realidad que a todos/as nos afecta e influir decididamente sobre las políticas y la "clase" política. La desobediencia civil, como señala Marcone (2009), constituye un resorte fundamental del dispositivo simbólico de los Estados democráticos y activa dos de los principios legitimadores del orden democrático: la soberanía popular y el reconocimiento mutuo del derecho a tener derechos. 
En España se visualizan las protestas a través de diferentes formas o performances (huelgas, encadenamientos, caceroladas, concentraciones ciudadanas, motines, abrazos y desnudos colectivos, manifestaciones, toma de edificios, impedimentos a desahucios, marchas, piquetes, grito mudo, agitando manos, etcétera) orientados a sensibilizar y ejercer influencia política, social y económica. Hemos pasado del silencio a la red y de la red a la calle (15M, Democracia real YA, Juventud sin futuro, Anonymus, \#no les votes, democracia, Movimiento, \#ARv12M, etcétera).

En este sentido, Tilly (2008), Tarrow (1997) y Tilly Tarrow (2006) señalan que los performances son las formas relativamente familiares y estandarizadas con las que un conjunto de actores realizan clamores colectivos hacia otro conjunto de actores políticos. Según Tilly (2008), éstas son, en parte, el resultado de procesos de innovación y aprendizaje. Los actores van registrando la eficacia y/o la adecuación de las acciones de protesta que desarrollan, adaptándolas, mejorándolas en cuanto a los recursos e iniciativas necesarias para su desarrollo, la división de tareas, la necesidad de implicar a otros, etc; si se quiere, aprendiendo cómo resolver los dilemas de movilización y coordinación que supone la acción colectiva que pretenden desarrollar, hasta asignarles una denominación compartida que, en su repetición, es reconocida por otros.

\section{La participación en el Sistema Autonómico de Servicios Sociales en España}

La Constitución Española de 1978 realiza un mandato a los poderes públicos a fin de promover las condiciones para que la libertad y la igualdad del individuo y de los grupos en que se integran sean reales y efectivas, remover los obstáculos que impidan o dificulten su plenitud y facilitar la participación de todos los ciudadanos en la vida política, económica, cultural y social. Todos estos principios orientan a desarrollar unas Políticas de Bienestar en las cuales la presencia de los ciudadanos no se limite a mera recepción de prestaciones, sino que incluya, además, la colaboración de los mismos en el establecimiento de los criterios de actuación de esta materia y en su propio desarrollo y evaluación.

En el desarrollo de estos derechos, las referencias legales básicas en el ámbito estatal son la Ley 7/1985, reguladora de las Bases de Régimen Local; el Real Decreto 2568/1986, del 28 de noviembre, por el cual se aprueba el Reglamento de Organización, Funcionamiento y Régimen Jurídico de las 
Entidades Locales y la Ley 57/2003, del 16 de diciembre de medidas para la Modernización del Gobierno Local. Esta última, en razón de nuestro análisis, constituye un intento por desarrollar "formalmente" los organismos y mecanismos de participación ciudadana en el ámbito local.

Con esta nueva normativa, el Estado español trata de incorporarse a las tendencias europeas que proponen reforzar las posibilidades de participación y de incidencia de los ciudadanos en el gobierno local para evitar o corregir el alejamiento de éstos de la vida pública. De manera concreta, contempla tres supuestas "novedades", específicamente la creación de distritos, del Consejo Social de la Ciudad y de una Comisión Especial de Sugerencias y Reclamaciones.

En la arquitectura del Estado Autonómico, los Servicios Sociales ${ }^{1}$ son competencia exclusiva de las Comunidades Autónomas, por lo tanto es preciso "acercarse" a ellas para el análisis de la participación ciudadana en los Servicios Sociales. El conjunto de leyes autonómicas de éstos contemplan, aunque a diferentes niveles, el principio de "participación ciudadana" o "cívica”, mediante la creación de mecanismos y cauces con los cuales impulsar la participación en la gestión del denominado "Sistema Público de Servicios Sociales", así como en la planificación, seguimiento, control y evaluación de los centros, planes y programas sociales.

Las leyes más recientes identifican la promoción de la participación como finalidad y/o principio rector del sistema (Ley de Cantabria 2/2007, ${ }^{2}$ Ley 5/2009 de Aragón, ${ }^{3}$ Ley 12/2008 del País Vasco, ${ }^{4}$ Ley 14/2010 de Castilla-La Mancha, ${ }^{5}$ Ley 16/2010 de Castilla y León ${ }^{6}$ ) objetivo (Ley Foral

1 Entre el catálogo de competencias de las CC.AA. se encuentran las relativas a la asistencia social (148.1.20).

2 Ley de Cantabria 2/2007, del 27 de marzo de Derechos y servicios sociales - B.o.C. núm.: 66 del 3 de abril.

3 Ley 5/2009, del 30 de junio, de Servicios sociales de Aragón - B.O.A. núm.: 132 del 10 de julio.

4 Ley 12/2008, del 5 de diciembre, de Servicios sociales del País Vasco - B.O.P.V. núm.: 246 del 24 de diciembre.

5 Ley 14/2010, del 16 de diciembre, de Servicios sociales de Castilla-La Mancha - B.O.E. núm.: 38 del 14 de febrero de 2011.

6 Ley 16/2010, del 20 de diciembre, de Servicios sociales de Castilla y León - B.O.E. núm.: 7 del 8 de enero de 2011. Bocyl. núm.: 244 del 21 de diciembre de 2010 y corrección de errores en BOCYL, núm.: 23 del 3 de febrero de 2011. 
15/2006; $;^{7}$ Ley 12/2007 de Cataluña; ${ }^{8}$ Ley 13/2008 de Galicia; ${ }^{9}$ Ley 5/2009 de Aragón; Ley 4/2009 de las Illes Baleares; ${ }^{10}$ Ley $7 / 2009$,de la Rioja, ${ }^{11}$ Ley 14/2010 de Castilla-La Mancha), prestación (Ley del Principado de Asturias 1/2003, ${ }^{12}$ Ley 14/2010 de Castilla-La Mancha) de las políticas de servicios sociales, competencia municipal y/o función específica de los servicios sociales de base.

Todas ellas contemplan la creación de mecanismos que canalicen la participación ciudadana, así como los derechos y deberes de las personas usuarias de centros, servicios y programas, directamente de manera individual o a través de entidades sociales representativas (no lucrativas, iniciativa y voluntariado). Ambos ejercicios de ciudadanía se plasman, especialmente, en las carteras de servicios de las leyes promulgadas a partir de 2005 que refuerzan "formalmente" la protección de las personas usuarias, con un principio de garantía de la participación y una descripción detallada de derechos y deberes, entre los que se identifica la participación de las personas como agentes de su propio cambio y de los grupos y entidades de la sociedad civil en el funcionamiento de los servicios sociales.

La participación ya estaba presente en las primeras leyes de servicios sociales de los años ochenta y noventa (y en su desarrollo normativo posterior), la novedad radica en su extensión a las carteras/catálogos de servicios, su vinculación a la calidad y a los derechos y deberes de las personas usuarias y profesionales, y al fortalecimiento de la iniciativa social en la provisión de servicios y libre elección del consumidor en la creciente externalización/privatización de los mismos.

El análisis de contenido normativo del conjunto de leyes autonómicas de servicios sociales en España ha permitido caracterizar el mapa de meca-

7 Ley Foral 15/2006, del 14 de diciembre, de Servicios sociales - B.O.E. núm.: 27 del 31 de enero.

8 Ley 12/2007, del 11 de octubre de Cataluña - B.O.E. núm.: 266 del 6 de noviembre.

9 Ley 13/2008, del 3 de diciembre, de Servicios sociales de Galicia - D.o.G. núm.: 245 del 18 de diciembre.

10 Ley 4/2009, del 11 de junio, de Servicios sociales de las Illes Baleares - B.O.B.B. núm.: 89 del 18 de junio.

11 Ley 7/2009, del 22 de diciembre, de Servicios sociales de la Rioja - B.O.R.: del 28 de diciembre.

12 Ley del Principado de Asturias 1/2003, del 24 de febrero, de Servicios sociales - B.o.P.A. del 8 de marzo. 
nismos y cauces de participación ciudadana en el ámbito autonómico y local conforme se señala a continuación:

- Mecanismos regionales de coordinación interadministrativa e interdepartamental para elaborar la planificación autonómica (planes estratégicos, mapas) y garantizar la participación de administraciones competentes (Observatorios y/o Consejos de Coordinación Interinstitucional o Interadministrativa).

- Comités técnicos de estudio de necesidades sociales y evaluación de la eficiencia y calidad de los servicios sociales y Comités de ética de los servicios sociales.

- Órganos regionales de participación, con funciones de asesoramiento, consulta y propuesta, y cuyo objeto es articular la participación y contribuir al mejor desarrollo, calidad y eficacia de los servicios sociales (Consejos Autonómicos).

- Órganos regionales asesores y consultivos en asuntos relativos a la atención a la dependencia (Comités Consultivo de Atención a la Dependencia).

- Órganos colegiados sectoriales creados al efecto por sectores de la acción social.

- Órganos provinciales, forales o insulares departicipación, orientadas al asesoramiento, consulta y propuesta y adscritos a la Diputación Provincial/ Foral/Insular (Consejos de Servicios Sociales).

- Mecanismos locales de participación en servicios sociales (Consejos Locales).

- Fomento de la participación de la iniciativa social en los servicios sociales. En este aspecto nos encontramos con leyes que diferencian la iniciativa social lucrativa (iniciativa mercantil) de la no lucrativa (iniciativa social). En otras, dicha diferenciación no existe, reconociendo por igual al derecho de participar en la creación de centros y servicios y únicamente ante análogas condiciones de calidad, eficacia y costes, darán prioridad a la colaboración con entidades de iniciativa social sin ánimo de lucro. En cuanto a las modalidades de colaboración se reconocen fórmulas como: concertación, convenios y acuerdos y subvenciones.

Del análisis de contenido normativo podemos constatar que son escasas las leyes y, en su caso, de manera ambigua las que señalan que podrá articularse a través del movimiento asociativo y mediante los procesos participativos. En cuanto a la participación de las personas usuarias, las leyes de "segunda" y "tercera" generación les atribuyen, al menos formalmente, un papel más ac- 
tivo, en concreto participar en todas las decisiones que les afecten de forma directa o indirecta, individual o colectiva. Todas las entidades y centros de servicios sociales deberán contar con procedimientos de participación democrática de las personas usuarias, o de sus representantes legales, de acuerdo con lo que se determine reglamentariamente; a tal efecto establecen como mecanismo el Consejo de usuarios.

Resulta de interés indicar que los derechos y deberes de las personas usuarias que incorporan las recientes leyes son una clara influencia de la Ley 39/2006, ${ }^{13}$ de Promoción de la Autonomía Personal y Atención a las personas en situación de dependencia (LAPAD), aunque en este sentido las evaluaciones sistemáticas de la aplicación de la citada ley muestran que la importancia concedida a la persona usuaria/beneficiaria respecto a la toma de decisiones en el proceso de diagnóstico e intervención social y su vinculación a criterios de calidad es más "formal/virtual" que real.

Atendiendo a recientes investigaciones sobre la materia (Gallego y Subirats, 2011), se constata que persiste en el imaginario la perspectiva tradicional asistencial y benéfica de los servicios sociales, asociados a la pobreza económica y a grupos de riesgos, comprobándose, además, una visión negativa de los servicios sociales, una inadecuada e insuficiente oferta de servicios en relación con las necesidades de la población (intensidad de cobertura), una dependencia excesiva de la agenda mediática y pública que puede ocasionar una errónea priorización de la intervención pública (extensión) más centrada en el populismo. Una heterogeneidad territorial en extensión e intensidad de cobertura que tiende a una insuficiente financiación Esta arbitrariedad y clientelismo político lo observamos de igual forma en la oferta de oportunidades e impacto real en las políticas de participación.

Por último, cabe destacar la creciente preocupación de las administraciones públicas por la sostenibilidad del sistema evidenciado en su inclusión en las últimas leyes y concebida como financiación suficiente del sistema para asegurar su estabilidad y continuidad en el tiempo y provocando, entre otros, la expansión del mercado y del tercer sector por la vía de la prestación de servicios y la internalización del coste de la protección a la dependencia por la vía del copago y la atención familiar informal, característico del modelo social europeo de atención a la dependencia y que ésta ha tenido consecuencias en la configuración del sistema de servicios sociales.

13 Ley 39/2006, de 14 de diciembre, de Promoción de la Autonomía Personal y Atención a las personas en situación de dependencia, publicada en el B.O.E. núm.: 299 del 15 de diciembre de 2006. 


\section{Caracterización y tipología de mecanismos de participación en el contexto regional}

Los mecanismos de participación ciudadana mayoritarios en las políticas de servicios sociales municipales son los siguientes: a) estructuras de participación de base asociativa o Consejos; ${ }^{14}$ b) organismos públicos autónomos desconcentrados o Institutos; ${ }^{15}$ y c) mecanismos no formales de participación de base asociativa. ${ }^{16}$

En el estudio empírico realizado en el contexto de la Región de Murcia y atendiendo a la anterior tipología, constatamos que de los 45 Ayuntamientos de la Región, sólo 11 de ellos $(24,4 \%)$ disponen de mecanismos formales de desconcentración y/o participación en materia de bienestar social o servicios sociales en sentido amplio (general o sectorial), aunque son ocho $(17,7 \%)$ los Ayuntamientos que en la práctica han puesto en marcha los citados órganos participativos. De éstos, dos de ellos disponen únicamente de Consejos Sectoriales pero no de carácter general, y otros tres abarcan un objeto más amplio al de los servicios sociales propiamente dicho, al ocuparse de diferentes áreas de la actividad pública de la Corporación Local (Consejo Municipal de Participación Ciudadana y de ONG). Por tanto, son cinco los Ayuntamientos que en la Región de Murcia disponen de Consejo (tres) o Instituto Municipal de Servicios Sociales (dos), de carácter general y que funcionan en la actualidad, representando a tan sólo el 11,1\% de las Corporaciones Locales de la Región.

Por otra parte, las cinco Mancomunidades de Servicios Sociales - existentes hasta 2007-, como Entidades Locales de carácter supramunicipal con

14 Se caracterizan por ser consultivos, de ámbito territorial y/o sectorial, de carácter extensivo y facilitadoras de información, asesoramiento y captación de propuestas.

15 Disponen de personalidad jurídica propia, delegación de competencias en servicios sociales generales y/o sectoriales y con cierta autonomía en la toma de decisiones y la gestión económico-administrativa y técnica del área. De esta forma, combinan: adopción de decisiones, ejecución/gestión de acuerdos y participación.

16 Dimensión informal del ejercicio de facilitación. A través de reuniones periódicas protagonizadas por técnicos de servicios sociales con organizaciones del territorio y técnicos de otros sistemas. La iniciativa, convocatoria, agenda y organización se realiza a través de los directores/coordinadores de los Centros de Servicios Sociales, en ocasiones sin respaldo sobre los asuntos tratados y acuerdos alcanzados en ellas. Entre sus fines se encuentra: ofrecer información, detectar necesidades, solicitar participación, promover la cooperación interasociativa y generar redes y protocolos de colaboración técnica interinstitucional. 
competencias "delegadas" en materia de servicios sociales por parte de los Ayuntamientos que la integran, gestionan directamente los Servicios Sociales de Atención Primaria de 23 Ayuntamientos, lo que representa más del $50 \%$ de los Ayuntamientos de la Región de Murcia. Ninguna de ellas optó, ni en su creación ni en su desarrollo hasta la actualidad, por la fórmula desconcentrada de gestión como es el Instituto de Servicios Sociales ni han impulsado en su seno la creación de Consejos Territoriales y/o Sectoriales de participación ciudadana en materia de servicios sociales.

Respecto de los Consejos Sectoriales, son seis los Ayuntamientos que los han puesto en marcha, representando al 13,33\% de los Entidades Locales existentes en la Región. Se encuentran en primer lugar los de Mujer y Personas Mayores, los cuales existen en cuatro Ayuntamientos. Concretamente, uno dispone de ambos, dos tienen Consejos de Mujer (Igualdad de Oportunidades y otros vinculado con la Violencia contra las Mujeres) y otro de personas mayores. Atendiendo a otros colectivos, dos Ayuntamientos disponen de Consejos de Inmigración y otros dos están centrados en las Drogodependencias.

Por consiguiente, únicamente cinco Ayuntamientos disponen de Consejos Municipales (o de Participación Ciudadana) y Sectoriales de manera simultánea. En cuanto a los Institutos, en la actualidad funcionan sólo en dos Ayuntamientos, representando el $4.4 \%$ de los municipios; aunque cabe precisar que dos Corporaciones Locales que en la actualidad disponen de Consejo Municipal, originariamente eran Institutos de gestión autónoma de los asuntos sociales municipales.

\section{Análisis de las oportunidades y limitaciones de los mecanismos de participación en la gestión de las políticas de servicios sociales locales}

A continuación se presentan los principales resultados del análisis de contenido y de las entrevistas en profundidad con los actores participantes en los mecanismos de participación regional y que nos permiten identificar las contribuciones, potencialidades y limitaciones más significativas de los Consejos Territoriales de Bienestar Social relacionadas con su capacidad para influir en los procesos de democratización en la construcción de las políticas sociales en el ámbito municipal.

\section{Modelo de participación institucional por y para la administración}

El análisis de la regulación normativa de los Consejos constata que éste otorga a la administración una enorme y flexible capacidad de control sobre agenda, 
actores participantes y procesos participativos. Los estatutos y reglamentos definen y determinan los niveles y asuntos sobre los que es posible participar, limitando de esta forma el marco y posibilidades reales de participación efectiva sobre los asuntos sociales públicos, a la vez de permitir al responsable político/técnico introducir en la agenda de las sesiones aquellas cuestiones que a éste le interesan.

De esta manera, los participantes se convierten en "consumidores-invitados" pero no protagonistas, pueden hacer valer su voz, pero filtrada en atención a su encaje en la agenda y estrategia de la acción política. Los mecanismos de participación no son percibidos por las organizaciones como un espacio propio, sino un punto de encuentro periódico con la administración local donde ésta les ofrece información acerca de las actuaciones realizadas o a realizar en política social. Para los técnicos, un marco donde rendir cuentas de su gestión de forma explícita y sistemática, percibiéndolos como carga de trabajo, control institucional y social, en mayor medida que como trabajo compartido. En definitiva, un modelo centrado en lo institucional y en procedimientos previstos y controlados por y para la administración.

\section{Participación asociativa e interferencias/interdependencias para la participación}

La composición y representatividad de los Consejos Municipales de Bienestar Social o Servicios Sociales tienen una base de participación, fundamentalmente, asociativa; con un claro protagonismo de las federaciones, fundaciones y asociaciones frente a los ciudadanos de manera individual, plataformas y entidades minoritarias; a la vez que se privilegia la representación sectorial a la territorial. Estas preferencias por las entidades sociales, fundamentalmente sectoriales (carácter especializado), como protagonistas en la participación es una pauta interaccional muy habitual en las políticas y experiencias participativas.

Se constata una progresiva burocratización, profesionalización, especialización funcional y dependencia del sector asociativo respecto de las administraciones públicas. Los procedimientos y condiciones de acceso al sectorializado y cada vez más concurrido y "exiguo" "fondo" público (contratos, acuerdos, subvenciones) puede llevar a las entidades a reducir su potencial de flexibilidad y capacidad de innovación, dado que al final la administración determina qué (centros y servicios), para qué (sentido y fines) y cómo se hace (modos de intervención) y a quién (colectivos y/o problemáticas diana) se han de prestar los servicios. 
Este dominio de la lógica pública, tanto político institucional como de prestación de servicios, somete a las organizaciones a dilemas, sobre su propia identidad, autonomía, ética y estrategias de intervención social. Este contexto genera entre las entidades relaciones de competencia - de lucha por posiciones de capacidad de disponer de poder administrativo - en mayor medida que de cooperación/redes/alianzas y que nos diferencian de modelos de bienestar más consolidados como el nórdico o el bismarkiano, donde estas entidades comparten responsabilidades con la Administración.

Por otra parte, la fragmentada y atomizada realidad asociativa dificulta procesos de representación y participación plural, especialmente en los municipios de mayor tamaño donde el tejido social es muy numeroso y diverso. Se observa que ciertas organizaciones sociales acaparan la representación social en múltiples foros participativos y se constatan contextos asimétricos de capacidad y oportunidad de acceder a los asuntos políticos. La desigualdad, por un lado, en el acceso a los espacios y oportunidades de participación de las organizaciones y, por otro, la capacidad y habilidad de los participantes en formular opiniones políticas, determina esta asimetría representativa de colectivos y problemáticas sujetos y objeto de la acción de los servicios sociales.

\section{Divergentes expectativas entre actores}

Se constata la existencia de una brecha entre los objetivos formales y reales que se alcanzan en la práctica, así como en la distinta percepción que los actores implicados disponen acerca de los objetivos reales - plasmados en sus "reglamentos"- y aquellos que estiman deberían cumplir (expectativas). Las entidades sociales implicadas los perciben como un instrumento para mantenerse informadas sobre los asuntos sociales municipales; intercambiar puntos de vista; informar a la administración de las acciones que realizan; disponer de un espacio de encuentro y colaboración entre entidades sociales que les permite conocer la existencia y el trabajo que cada una hace, coordinar servicios y actuaciones, y trasladar necesidades y reivindicaciones de colectivos.

Esta comunicación ha mejorado, concretamente en lo referente a la derivación de usuarios/as y el desarrollo de acciones concretas entre los Centros de Servicios Sociales y las organizaciones sociales. Éstas valoran muy favorablemente dichos mecanismos especialmente por la posibilidad de diálogo e intercambio de información con los profesionales de los servicios sociales. Para los técnicos supone un instrumento para detectar necesidades sociales, agilizar procesos y, en ocasiones, mejorar la coordinación, de las cuestiones 
cotidianas del Área (seguimiento de casos, altas y bajas en prestaciones económicas y servicios, información de proyectos y resultados).

Uno de los aspectos más visibles e importantes de la participación analizada es su competencia a la hora de determinar las formas de hacer; los aspectos metodológicos de las sesiones, las cuestiones y la manera en cómo éstas se abordan no facilitan la consulta, deliberación y elaboración de propuestas. El proceso participativo es percibido y considerado un trámite más administrativo que sustantivo.

\section{Agenda instrumental y opacidad}

Los asuntos tratados se relacionan con la presentación e información de servicios, proyectos actuaciones, presupuesto; normativas reguladoras de prestaciones y ayudas y casos. Por tanto, centrados en requerimientos técnicos y administrativos y no de carácter político, tales como la elaboración de diagnósticos, proyectos y toma de decisiones compartidas. A su vez, la superficialidad con la que son tratados genera confusión en la comprensión de la realidad compleja de estos fenómenos.

La gestión técnica/administrativa de los asuntos sociales adquiere carácter preferente en la agenda, organización, funcionamiento y dinámica de estos mecanismos. El proceso participativo es dirigido por el discurso técnico a partir de cuestiones decididas políticamente, desestimulando a la participación. La necesaria incorporación real del debate y la deliberación en los asuntos sociales requiere de "tiempos" y "lenguajes" que permitan el análisis y elaboración de propuestas por parte de las entidades y de manera previa a la celebración de las sesiones. Pero también es cierto que existen importantes limitaciones administrativas y técnicas cuando determinadas propuestas de centros, programas y proyectos deben atenerse a unos plazos marcados por órganos ajenos al Consejo, e incluso de la misma administración local o centro, con el fin de captar fondos autonómicos y/o estatales. Se constata que la ciudadanía en general y las organizaciones sociales no participantes desconocen la existencia y/o funcionamiento de los Consejos, dado que el proceso de formalización y funcionamiento no ha estado acompañado de actuaciones, previas y posteriores, suficientes de información, difusión, consulta, propuesta y debate. Esta insuficiente transparencia retroalimenta/contribuye a la arbitrariedad en la selección de actores y funcionamiento de los mecanismos, reproduciendo sesgos en la participación. 


\section{Predominio de la información y consulta no vinculante}

Los actores acuden a los Consejos con una información documentada y exhaustiva pero también sesgada y filtrada por quién y qué información y cómo se ofrece, lo cual determina la formación de las opiniones individuales y colectivas respecto de la realidad y alternativas que se presentan como objetivas y posibles. La información no es algo dado, está sometida a un proceso de construcción de datos e interpretaciones previos que son utilizados para definir la realidad y plantear cursos de acción posibles.

La consulta, en su caso, aparece como un "rito" democrático limitado a expresar, no siempre, la opinión acerca de lo que se pregunta, en el marco de las alternativas definidas como posibles. No conlleva deliberación sino una mera agregación de opiniones y, por tanto, más relacionada con una estrategia de conocer las posibilidades de éxito o fracaso de una determinada acción política y/o técnica - a realizar o realizada. Los temas y el cómo son planteados, no suelen tener opciones de modificación o el margen es muy reducido.

La escasa vinculación de los acuerdos y la percepción de no influir en la política social local de manera práctica conlleva que los miembros se sientan, en ocasiones, "invitados", "no participantes" - tal y como manifiestan- en el proceso de toma de decisiones reales apareciendo situaciones de absentismo (no asistencia a sesiones). Las organizaciones "enmudecen" adoptando un papel "ausente" e incluso de "conformismo" en las sesiones, utilizando otras vías más útiles para canalizar sus demandas, como se advirtió anteriormente, e influir en la toma de decisiones. Los ciudadanos están dispuestos a invertir su tiempo libre y otros recursos si su participación mejora su calidad de vida y la calidad de los servicios que ocupan.

Si la participación de los ciudadanos, por el contrario, no ofrece resultados visibles, los ciudadanos pierden interés. Así, los procesos de consulta que no llevan a ninguna parte y que solamente se ciñen al proceso de toma de decisiones ciertamente no incrementan la confianza ciudadana en sus gobiernos locales si, como nos señala Löffer (2004), las decisiones no se toman considerando la perspectiva de los ciudadanos.

\section{El buen gobierno de las políticas públicas de bienestar social en el ámbito local: intensificando la calidad democrática}

En coincidencia con las conclusiones de estudios sobre la democracia asociativa (Löffler, 2004; Montero, Font y Torcal, 2006; Colino y Del Pino, 2008; 
Navarro, 2008; Navarro, Cuesta y Font, 2009; Pastor, 2009, 2011), la política municipal de participación en los servicios sociales municipales conforma un modelo democrático que puede denominarse de "democracia de acceso", pues queda fuera la incorporación de la ciudadanía en la formulación efectiva de las políticas, no ya en la activación de la agenda, sino en la discusión en torno a los problemas que ésta debe o no debe incluir.

Se produce una división que "separa el conjunto de las cosas importantes, por un lado, de las cosas accesorias, por otro" (Gutiérrez, 2005: 180). Mientras, el discurso de la participación institucional se refiere a ésta como deliberación y discusión de los asuntos públicos, la realidad demuestra que los Consejos se ocupan de aspectos instrumentales, formales y secundarios, orientados a legitimar políticas institucionales y, en ocasiones, de los profesionales de los centros de servicios sociales.

Los Consejos e Institutos favorecen procesos de interacción entre los actores, pero no garantizan por sí mismos y de forma automática una democratización en la construcción de las políticas sociales en el ámbito local ni viceversa. De ahí que más allá de su regulación, requiere de un diseño, organización y funcionamiento que posibilite condiciones y oportunidades reales para acceder e influir en los procesos de toma de decisiones respecto de las políticas sociales.

Atendiendo al análisis desde los participantes de la investigación, podemos sistematizar las propuestas orientas a mejorar: a) el sistema de servicios sociales en el ámbito municipal y b) los mecanismos y prácticas participativas.

Aportaciones para impulsar mejoras en el sistema de servicios sociales en el ámbito municipal

10) Mayor autonomía normativa, económica y de gestión de la administración local en materia de servicios sociales de atención primaria. Transferir a las Corporaciones Locales el presupuesto y las competencias necesarias para una adecuada y autónoma gestión de los servicios y elaborar planes estratégicos en cada una de las Corporaciones Locales.

$2^{\circ}$ ) Implantar un sistema organizativo en la administración local que promueva las politicas públicas de manera participativa. Crear Concejalías de Participación Ciudadana que promuevan órganos de participación asociativa y de participación directa de los ciudadanos en las diferentes Áreas de gestión municipal; impulsar la elaboración de políticas sociales transversales desde la coordinación de las diferentes áreas de competencia municipal aten- 
diendo a la participación como elemento esencial para su configuración y alcanzar acuerdos políticos que eviten la instrumentalización partidista de los mecanismos.

$3^{\circ}$ ) Crear instrumentos normativos que regulen el derecho y los mecanismos participativos en la administración local. Impulsar medidas que garanticen la participación de las personas y entidades sociales en los procesos de toma de decisiones en los asuntos sociales públicos: promover Reglamentos Municipales de Participación Ciudadana, elaborar y aprobar Cartas de los Derechos de los Ciudadanos e incorporar las posibilidades de las nuevas tecnologías para innovar en la participación y crear Registros Municipales accesibles.

$\left.4^{\circ}\right)$ Universalizar la cobertura de Consejos territoriales y sectoriales de Servicios Sociales en todas aquellas Corporaciones Locales con competencia en la gestión de los Servicios Sociales de Atención Primaria. Crear Consejos Municipales de Servicios Sociales generales, generando estructuras intermedias de participación de carácter sectorial y territorial, ampliando la cobertura de participantes y vinculando acuerdos y propuestas de manera ascendente y descendente.

50) Desconcentrar y descentralizar la gestión de los Servicios Sociales Municipales en órganos intermedios participativos y en el territorio. Gestionar los Servicios Sociales de Atención Primaria desde organismos públicos autónomos. Los Institutos son instrumentos adecuados de desconcentración de la gestión de las competencias de servicios sociales en el ámbito municipal, favorecen la transparencia y agilidad en la gestión económica, técnica y administrativa de los asuntos sociales y permiten incorporar mecanismos participativos.

$\left.6^{\circ}\right)$ Mejorar la representatividad y diversidad de actores participantes en los Consejos e Institutos. Romper la dicotomía entre elevada representatividad social vs. eficacia en gestión de los asuntos públicos. Para ello se sugiere la articulación de Mesas de Trabajo o Consejos Sectoriales con competencias delegadas y que permitan la adopción de acuerdos en órganos intermedios representativos de cada colectivo o problemática social concreta que represente al conjunto de entidades y organizaciones vinculadas con cada sector de población. Considerando el vertiginoso crecimiento de las asociaciones y otras entidades sociales, parece conveniente incluir en los estatutos o reglamentos la flexibilidad de incorporar a nuevos miembros sin necesidad de modificar éstos, simplemente mediante acuerdos al respecto. Establecer condiciones y garantías de representación de los miembros elegidos al efecto. Se trata de que los representantes se comporten como tales: recogiendo previamente demandas y necesidades, y trasladándolas adecuadamente, informando de ma- 
nera continua. Incorporar en los Consejos e Institutos la diversidad social, integrando con mayor intensidad a grupos sociales que en la actualidad se encuentran infrarrepresentados.

$\left.7^{\circ}\right)$ Generar estrategias relacionales de mayor impacto en las politicas sociales locales. Clarificar objetivos y funciones de los Consejos e Institutos respecto de su incidencia directa en la política local, especificando los asuntos que ofrezcan un contenido real a estos mecanismos y, por tanto, motivar para participar. Se trata de innovar en el diseño e implementación de metodologías participativas que faciliten la participación real y evitar "caer" en la "inercia" de la no participación. Para ello se considera oportuno compatibilizar mecanismos de participación asociativa con formas que promuevan la participación directa; vitalizar el capital social local, socializar y educar para la participación, impulsar y apoyar el asociacionismo y movimientos cooperativos, promover relaciones asociativas, generar redes sociales locales, apoyar acuerdos estratégicos entre organizaciones sociales y la Administración Local; coordinar centros y servicios locales, fomentar la co-gestión con las organizaciones vecinales y sociales de centros municipales; optimizar recursos, impulsar medidas de cooperación social entre organizaciones y la Administración Local, dotar con recursos municipales a los órganos de participación ciudadana existentes, generar espacios de encuentro entre Administraciones, etcétera.

Aportaciones para profundizar en prácticas participativas orientadas a generar un diálogo significante en el ámbito local

Las dimensiones a considerar para intensificar la participación ciudadana en los procesos, prácticas, actores y resultados pueden sintetizarse del siguiente modo:

$\left.1^{\circ}\right)$ Preparación adecuada. Los contextos institucionales moldean significativamente los roles que adoptan los actores, los procesos y, por tanto, los resultados y la satisfacción de los actores. De ahí la necesidad de una coherencia en la organización municipal en escuchar y vincular los asuntos y acuerdos a la acción de gobierno. Por tanto, se precisa de: estructura administrativa, desarrollo normativo, recursos de apoyo a organización y desarrollo y voluntad política. Una responsabilidad definida, un liderazgo sólido que vincule la acción de gobierno local en su conjunto.

$\left.2^{\circ}\right)$ Planificación inclusiva y criterios de proceso. Supone acordar "reglas de juego", claridad en contexto, finalidad, propósitos, diseño en procesos y 
tareas. Deben cuidarse los ritmos, lugares de realización, y criterios y selección de actores a participar. Los mecanismos de participación existentes en un gobierno deben estar conectados entre sí con el fin de dar una visión integradora de ciudadanía. El proceso participativo debe satisfacer lo emocional (que los participantes y ciudadanía se sientan reconocidos, respetados y escuchados), el interés genuino de los participantes que obtienen resultados y sentir/percibir/objetivar que el procedimiento es legítimo, justo, valioso. Estas cuestiones requieren evitar la improvisación, la fatiga del diálogo y "simulacros".

$3^{\circ}$ ) Inclusión y diversidad de actores/territorios. Supone la incorporación equitativa de actores, no sólo desde la representatividad estadística, sino especialmente desde la integración de todas las posiciones/voces. Ello requiere la igualdad en consideración/reconocimiento en los debates desde una comunicación horizontalizada. Los criterios y procesos de selección de actores deben ser transparentes, evitando privilegiar a "grandes corporaciones", ignorar a opositores, o bien, privilegiar en exceso a "expertos”. La máxima inclusión/representatividad implica que la ciudadanía en general debe tener la posibilidad de acceder e implicarse en las decisiones políticas y no solamente las personas y grupos cualificados. No resultan eficaces los mecanismos y procedimientos que excluyen de los procesos de toma de decisión a las personas o grupos por falta del "capital" (social, cultural, económico, tecnológico).

40) Equipo dinamizador y dinámica facilitadora. Los facilitadores deben ser imparciales y cualificados, su tarea principal es cultivar una sensación compartida de apropiación, "empoderando" a la ciudadanía mediante un diálogo significante. Esto requiere promover la indagación, la transparencia comunicativa y la autorreflexión. Ingredientes que potenciarán la comunicación, generando un ambiente de confianza orientado a identificar coincidencias y diferencias argumentadas. En este sentido, cabe incidir en que participar no es sólo asistir a reuniones, sino apropiarse de los procesos de toma de decisiones desde los diferentes mecanismos y medios que disponemos para escuchar y dar salida a la voz de los ciudadanos individualmente considerados, como grupos no organizados y como entidades sociales.

50) Dinámica motivadora. Los mecanismos deben motivar y estimular para participar. Los participantes deben tener la sensación y certeza de que sus esfuerzos van en serio y que sus propuestas no se quedan en "papel mojado", sino que van a ser tomadas en consideración a la hora de tomar las respectivas decisiones. Las experiencias muestran el riesgo de utilizar los mecanis- 
mos para justificar decisiones adoptadas previamente, instrumentalizándose la opinión pública.

$\left.6^{\circ}\right)$ Colaboración y propósito compartido. Supone trabajar en la planificación, recogida y presentación de información, etc., evitando promover opciones principales y secundarias.

$7^{\circ}$ ) Apertura y aprendizaje. Ayudar a escucharse, explorar nuevas ideas sin restricciones, generar nuevas opciones; mediante un espacio de aprendizaje, distensión, autorreflexión. Para ello es importante cuidar ritmos, generar momentos de inflexión que permita a los participantes mostrarse vulnerables y hacerse vulnerables y permeables al resto de los actores.

$\left.8^{\circ}\right)$ Funcionamiento deliberativo. El proceso metodológico de las sesiones debe promover la deliberación. Los participantes no deben discutir sobre la base de la información que traen, sino que se debe disponer de información extensa y diversa sobre los pros y contras, los diferentes intereses y opciones de la cuestión a tratar. Se trata de plantear todos los aspectos de un tema, hacerlo transparente, presentar los diferentes intereses, las soluciones alternativas, los costes y las repercusiones sobre la resolución de otros temas (ranking de prioridades) como sobre las personas afectadas. Es preciso transferir esas capacidades a la ciudadanía para que ésta disponga del capital y poder para adoptar decisiones autónomas. Pero el "input" de información no se debe confundir con su utilización para conseguir el apoyo de la ciudadanía a una decisión adoptada por la Administración.

90) Transparencia y confianza. La información debe ser accesible, multidireccional, policéntrica y desde diferentes medios. Se trata de generar procesos claros y flexibles tanto en la información como en la recogida de opciones, análisis de las mismas y generación de alternativas analizadas desde la viabilidad y la perspectiva de futuro.

10 $0^{\circ}$ Apoyo del bien general. Los participantes deben apoyar el bien común, es decir, la adopción de decisiones importantes para el futuro de la comunidad y no solamente de determinados grupos. Ello requiere de un diagnóstico comunitario basado en criterios de priorización adoptados de manera participativa.

110) Impacto y acción. Asegurarse de que cada esfuerzo participativo tiene potencial real, lo cual implica mecanismos de participación interconectados, adecuados, efectivos y capacitantes. Los participantes deben evidenciar que su participación es significativa, influye, impacta una sensación de apropiación real sobre procesos, tareas y decisiones. 
120) Compromisos sostenidos-cultura participativa. Los procesos participativos deben vincularse entre sí con el fin de ir generando una cultura participativa en el contexto local que involucre a todos los ámbitos de la vida cotidiana y agentes socializantes, para generar una ciudad participativa.

El análisis constata que los procesos participativos deben:

- Ocuparse de la complejidad: social (perspectivas e intereses diversos), dinámica (causas y efectos sin tiempo y espacio) y generativa (futuro incierto/indeterminado).

- Coordinar significados/entendimientos diversos: confianza y aceptación mutua, lo que implica transparencia de procesos y resultados.

- Producir innovación: respuestas novedosas, diferentes y tangibles a la ciudadanía.

- Posibilitar la deliberación: dialogar, sistematizar opiniones, encuadrar opciones alternativas y adoptar decisiones firmes acordadas.

- Diálogo sostenido: producir procesos/resultados sostenibles a largo plazo.

- Generar capacidades: empoderar, capacitar, crear grupos de trabajo, generar y apoyar líderes y su rotación, etcétera.

En resumen, la transparencia e innovación real y efectiva de la participación ciudadana en el diseño, gestión y evaluación de las políticas de servicios sociales se configura como elemento esencial para generar/reforzar/reconstruir la calidad democrática en el nivel territorial y organizacional más próximo. Su incorporación permitirá mejorar la eficacia y eficiencia de las políticas públicas y la prestación de los servicios sociales, y adoptar decisiones significativas y vinculantes para los actores sociales y usuarios de centros/servicios, provocando una progresiva vitalización del capital social de los municipios y organizaciones sociales donde trabajamos desde las sinergias transaccionales.

\section{Bibliografía}

Amnistía Internacional (2011), Derechos a la intemperie. Obstáculos para hacer valer los derechos económicos, sociales y culturales en España, Madrid: Amnistía Internacional.

Aguirre,J. F. (2009), "Ciudadanía hermenéutica. Un enfoque que rebasa el multiculturalismo de la aldea global en la sociedad del conocimiento", en Andamios. Revista de Investigación Social, agosto, vol. 6, núm. 11, México, D.F.

Alguacil, J. (2008), "Espacio público y espacio político. La ciudad como el lugar para las estrategias de participación”, en Revista Académica de la Universidad Bolivariana, vol. 7, núm. 20, Santiago, Chile.

Bobbio, N. (2003), Teoría general de la politica, Madrid: Trotta. 
Blondiaux, L. (2008), Le nouvel esprit de la démocratie. Actualité de la démocratie participative, París: Seuil y La République des Idées.

Chambers, S. (2003), "Deliberative Democratic Theory", en Annual Review of Political Science, núm. 6, Palo Alto, Estados Unidos de América.

Colino, C. y E. Del Pino (2008), "Democracia participativa en el nivel local: debates y experiencias en Europa”, en Revista catalana de dret públic, núm. 37, Barcelona, España.

Cunill, N. (1991), La participación ciudadana, Caracas: Centro Latinoamericano de Administración para el Desarrollo.

Cunill, N. (1997), Repensando lo público a través de la sociedad. Nuevas formas de gestión pública y representación social, Caracas: Nueva Sociedad, Centro Latinoamericano de Administración para el Desarrollo.

Espinosa, M. (2009), "La participación ciudadana como una relación socio-estatal acotada por la concepción de democracia y ciudadanía”, en Andamios. Revista de Investigación Social, abril, vol. 5, núm. 10, México, D.F.

Font, J. (2001), Ciudadanos y decisiones politicas, Barcelona: Ariel.

Gallego, R. y J. Subirats [dirs.] (2011), Autonomies i desigualtatas a Espanay: percepciones, evolució social $i$ politiques de benestar, Barcelona: Institut d'Estudis Autonómics, Generalitat de Catalunya.

Ganuza, E. y F. J. Frances (2008), “¿A qué llamamos participar en democracia?: Diferencias y similitudes en las formas de participación”, en Revista Internacional de Sociología, vol. LXVI, núm. 49, Madrid, España.

Giddens, A. (2000), Más allá de la izquierda y la derecha, el futuro de las políticas radicales, Madrid: Cátedra.

Gutiérrez, M. (2005), La participación en los servicios públicos de bienestar, Madrid: CES.

Hamzaoui, M. (2006), "La gestión territorial de los problemas sociales", en C. Larivière y J. M. Gorvil [coord.], "La gestión de lo social", en Revista Internacional de Politicas Sociales en Europa, vol. 20, Barcelona: Hacer.

Harms, H. y S. Pereyra (2006), "La necesidad de repensar la democracia”, en Revista de Ciencias Sociales Sistema, vol. 193, Madrid, España.

Held, D. (2001), Modelos de democracia, Madrid: Alianza.

INAP (2008), Evaluación de la participación pública en la elaboración de las políticas públicas, Madrid: Ministerio de Administraciones Públicas.

Jorba, L. (2009), Deliberación y preferencias ciudadanas, Madrid: Centro de Investigaciones Sociológicas.

Löffler, E. (2005), “Experiencias internacionales de participación ciudadana en Europa”, en 1er. Congreso Internacional de Democracia Participativa, Madrid.

Marcone, J. (2009), "Las razones de la desobediencia civil en las sociedades democráticas”, en Andamios. Revista de Investigación Social, abril, vol. 5, núm. 10, México. D.F.

Máiz, R. (2000), "Democracia participativa. Repensar la democracia como radicalización de la política”, en Metapolitica, abril-junio, vol. 5, núm. 18, México.

Montero, J. R., J. Font y M. Torcal [eds.] (2006), Ciudadanos, asociaciones y participación en España, Madrid: Centro de Investigaciones Sociológicas. 
Convergencia Revista de Ciencias Sociales, núm. 68, 2015, Universidad Autónoma del Estado de México

Naser, A. y G. Concha (2011), El gobierno electrónico en la gestión pública, Santiago de Chile: Instituto Latinoamericano y del Caribe de Planificación Económica y Social (ILPES) de CEPAL.

Navarro, C. J., M. Cuesta y J. Font (2009), ¿Municipios participativos? Participación politica y ciudadanía en ciudades medias españolas, Madrid: Centro de Investigaciones Sociológicas.

Navarro, C. (2008), Participación Local, Madrid: Centro de Investigaciones Sociológicas.

Navarro, C. (2011), Comunidades locales y participación politica en España, Madrid: Centro de Investigaciones Sociológicas.

Pares, M. [coord.) (2009), Participación y calidad democrática. Evaluando las nuevas formas de democracia participativa, Barcelona: Ariel.

Pasquino, G. (1999), La democracia exigente, México: Fondo de Cultura Económica.

Pastor, E. (2009), Participación ciudadana y gestión de politicas sociales municipales, Murcia: Editum.

Pastor, E. (2011), "Impact, effectiveness and sustainability of social policies and local democracy through the social involvement", en Revista de Cercetare si Interventie Sociala, vol. 35, Iasi, Romania.

Pastor, E. y M. Sánchez (2014), "Analysis and Impact of the Economic Crisis and Regulatory Changes in the Needs and Benefits System Municipal Social Services: Analysis Case of Murcia-Spain”, en Revista de Cercetare si Interventie Sociala, vol. 47, Iasi, Romania.

Putnam, R. D. (2011), Para que la democracia funcione, Madrid: Centro de Investigaciones Sociológicas.

Rodríguez, G. et al. (2005), Actores sociales y reformas del bienestar, Madrid: Centro Superior de Investigaciones Científicas.

Sauca, J. M. y M. I. Wences (2009), "Participación ciudadana y diversidad cultural: La Comision Bouchard-Taylor", en Andamios. Revista de Investigación Social, abril, vol. 5 , núm. 10, México, D.F.

Sintomer, Y. y E. Ganuza (2011), Democracia participativa y modernización de los servicios públicos: una investigación sobre las experiencias de presupuestos participativos en Europa, TNI, La Découverte, Ámsterdam, París.

Subirats, J. (2007), Los Servicios Sociales de Atención Primaria ante el cambio social, Madrid: Ministerio de Trabajo y Asuntos Sociales.

Tarrow, S. (1997), El Poder en Movimiento: los nuevos movimientos sociales, la acción colectiva y la politica, Madrid: Alianza Universidad.

Tilly, C. (2008), Contentious Performances, Cambridge: Cambridge University Press.

Tilly Y. y S. Tarrow (2006), Contentious Politics, Boulder (Co): Paradigm Publishers.

Vallespín, F. (2000), El futuro de la politica, Madrid: Taurus.

Warren, M. E. (2001), Democracy and association, Princeton: Princeton University Press. 
Enrique Pastor Seller. PhD. Doctor en Sociología. Profesor Titular de la Universidad de Murcia, España, Facultad de Trabajo Social, Departamento de Sociología y Trabajo Social. Director del grupo de investigación: “Trabajo Social y Servicios Sociales”. Líneas de investigación: bienestar social, políticas sociales, participación ciudadana, servicios sociales municipales, educación superior, desarrollo local, mediación. Publicaciones recientes: Pastor, E., G. Támez y K. Sáenz [eds.], Gobernabilidad, ciudadania y democracia participativa: análisis comparado España-México, Madrid: Dykinson (2014); Pastor, E. y M. Sánchez, "Analysis and Impact of the Economic Crisis and Regulatory Changes in the Needs and Benefits System Municipal Social Services: Analysis Case of Murcia-Spain”, en Revista de Cercetare si Interventie Sociala, 47 (2014); Pastor, E., "La Mediación como servicio público de la Administración local en las sociedades relacionales contemporáneas", en Revista Brasileira de Gestão e Desenvolvimento Regional, vol. 10, núm. 1 (2014).

Recepción: 5 de octubre de 2014.

Aprobación: 21 de enero de 2015. 
